\title{
Determination of kinetic parameters of decomposition of tetrahydropyrimidine derivatives
}

\author{
Kapil Bhesaniya, Ashish Patel, Shipra Baluja* \\ Department of Chemistry, Saurashtra University, Rajkot-360 005, Gujarat, India \\ *E-mail address: shipra_baluja@rediffmail.com
}

\begin{abstract}
Some new tetrahydropyrimidine derivatives have been synthesized and their structural determination was done by IR, NMR and mass spectral data. Thermal analysis of these synthesized dihydropyrimidinthiones has been carried out by TGA and DSC techniques. From the thermograms, various kinetic parameters such as order of degradation $(n)$, energy of activation $(E)$, frequency factor $(A)$ and entropy change $(\Delta S)$ have been evaluated. Further, thermal stability of tetrahydropyrimidine have been determined which is found to depend on the type of substituent present in the compounds.
\end{abstract}

Keywords: Tetrahydropyrimidine derivative; TGA, DSC; kinetic parameter

\section{INTRODUTION}

Synthesis of tetrahydropyrimidine and their derivatives is of high interest in organic chemistry. The pyrimidine fragment is present in various biologically active compounds, many of which have been found use in medical practice ${ }^{1,2}$. Thus, recently, much attention has been paid to derivatives of pyrimidine, including their hydrogenation products. This class of compounds displays wide ranges of biological and pharmacological properties such as antiinflammatory $^{3-5}$, analgesic ${ }^{6,7}$, antitumor ${ }^{8,9}$, antidepressant ${ }^{10}$, antibacterial and antifungal effects ${ }^{11-13}$. For the complete development of a new drug, thermal analysis has many applications ${ }^{14,15}$. The information obtained regarding the compounds under study is useful for the initial chemical research phase ${ }^{16}$. In the chemical research phase, thermal analysis plays an important role. The purity of the compound, the compounds ability to be able to exist in various crystalline forms as well as to characterize polymorphs and other forms of solid state should be investigated. It also makes possible to determine optimum conditions of storage of drugs and to define the parameters of technological processes, which can be used without loss of specific physicochemical properties of a drugs ${ }^{17,18}$. The application of these compounds in pharmaceutical field prompted us to study their thermal stability. In present study, some new tetrahydropyrimidines have been synthesized and characterization of these synthesized compounds is done by IR, NMR and mass spectral data. The thermal analysis of some new synthesized tetrahydropyrimidine derivatives has been done by DSC and TGA techniques. 


\section{EXPERIMENTAL SECTION}

Synthesis of 2,4-diamino-6-phenyl-1,4,5,6-tetrahydropyrimidine-5-carbonitrile (PAB-101 - PAB-110): A solution of substituted aldehyde (0.01 mole), malenonitrile (0.01 mole), freshly prepared sodium ethoxide $(30 \mathrm{ml})$ and guanidine hydrochloride $(0.01 \mathrm{~mole})$ was refluxed for 12 hours. The reaction mixture was poured into crushed ice and the solution was neutralized with aqueous $\mathrm{HCl}$ solution. The product was extracted using chloroform.

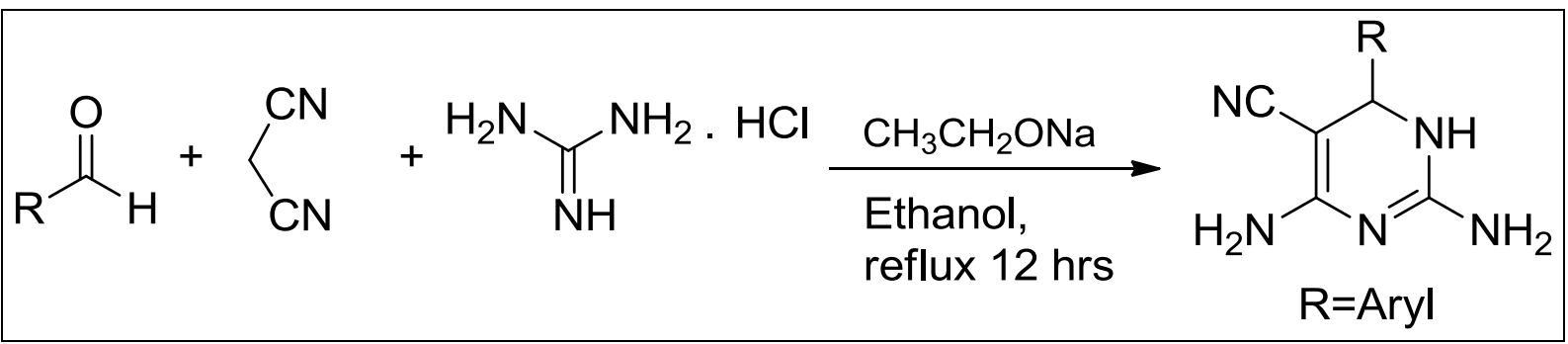

The formation of the compounds was checked by thin-layer chromatography and accomplished on 0.2-mm pre coated plates of silica gel G60 $\mathrm{F}_{254}$ (Merck). Visualization was made with UV light (254 and $365 \mathrm{~nm}$ ) or with an iodine vapor.

The melting point of all the synthesized compounds was determined in open capillary tubes and was uncorrected.

The characterization of all these compounds was done by IR, NMR and mass spectral data. The IR spectra were recorded on Shimadzu FT-IR-8400 instrument using KBr pellet method. The Mass spectra were recorded on Shimadzu GC-MS-QP-2010 model using direct inlet probe technique. ${ }^{1} \mathrm{H}$ NMR and ${ }^{13} \mathrm{C}$ NMR was determined in DMSO solution on a Bruker Ac $400 \mathrm{MHz}$ spectrometer.

The physical constants of all the synthesized compounds are given in Table 1.

Table 1. Physical properties of substituted tetrahydropyrimidine.

\begin{tabular}{|c|c|c|c|c|c|}
\hline Code & $\mathbf{R}$ & M.F & Yield (\%) & $R_{f}$ value & m. p. $\left({ }^{\circ} \mathrm{C}\right)$ \\
\hline PAB-101 & $\mathrm{C}_{6} \mathrm{H}_{5^{-}}$ & $\mathrm{C}_{11} \mathrm{H}_{13} \mathrm{~N}_{5}$ & 78 & 0.68 & $141-143$ \\
\hline PAB-102 & $\mathrm{C}_{6} \mathrm{H}_{4}-\mathrm{CH}=\mathrm{CH}-$ & $\mathrm{C}_{13} \mathrm{H}_{15} \mathrm{~N}_{5}$ & 60 & 0.73 & $112-114$ \\
\hline PAB-103 & $3-\mathrm{Cl}, \mathrm{C}_{6} \mathrm{H}_{4}-$ & $\mathrm{C}_{11} \mathrm{H}_{12} \mathrm{ClN}_{5}$ & 68 & 0.44 & $153-155$ \\
\hline PAB-104 & $4-\mathrm{Cl}, \mathrm{C}_{6} \mathrm{H}_{4-}$ & $\mathrm{C}_{11} \mathrm{H}_{12} \mathrm{ClN}_{5}$ & 66 & 0.46 & $162-164$ \\
\hline PAB-105 & $4-\mathrm{F}, \mathrm{C}_{6} \mathrm{H}_{4-}$ & $\mathrm{C}_{12} \mathrm{H}_{15} \mathrm{FN}_{5}$ & 62 & 0.52 & $128-130$ \\
\hline PAB-106 & $4-\mathrm{OCH}_{3}, \mathrm{C}_{6} \mathrm{H}_{4-}$ & $\mathrm{C}_{12} \mathrm{H}_{15} \mathrm{~N}_{5} \mathrm{O}$ & 84 & 0.42 & $116-118$ \\
\hline PAB-107 & $3-\mathrm{NO}_{2}, \mathrm{C}_{6} \mathrm{H}_{4}-$ & $\mathrm{C}_{11} \mathrm{H}_{12} \mathrm{~N}_{6} \mathrm{O}_{2}$ & 60 & 0.62 & $141-143$ \\
\hline PAB-108 & $\begin{array}{c}3-\mathrm{OCH}_{3} \\
4-\mathrm{OHC}_{6} \mathrm{H}_{4}^{-}\end{array}$ & $\mathrm{C}_{12} \mathrm{H}_{15} \mathrm{~N}_{5} \mathrm{O}_{2}$ & 74 & 0.34 & $132-134$ \\
\hline РAB-109 & $4\left(\alpha-\mathrm{C}_{4} \mathrm{H}_{3} \mathrm{O}\right)-$ & $\mathrm{C}_{9} \mathrm{H}_{11} \mathrm{~N}_{5} \mathrm{O}$ & 82 & 0.36 & $72-74$ \\
\hline PAB-110 & $4-\mathrm{OH}-\mathrm{C}_{6} \mathrm{H}_{4-}$ & $\mathrm{C}_{11} \mathrm{H}_{13} \mathrm{~N}_{5} \mathrm{O}$ & 56 & 0.30 & $176-178$ \\
\hline
\end{tabular}




\section{SPECTRAL DATA}

2,4-diamino-6-phnyl-1,4,5,6-tetrahydropyrimidine-5-carbonitrile (PAB-101).

IR (KBr): 3151(N-H str), 3093(Ar, C-H str), 2943(C-Hstr), 2867(C-H str), 2245(C $\equiv \mathrm{N}$ str), 1610(Ar, C $=\mathrm{C}$ str), 1519(Ar, $\mathrm{C}=\mathrm{C}$ str), 1512(Ar, $\mathrm{C}=\mathrm{C}$ str), 1490(C-H ben), 1427(C-H ben), 1377(C-H ben), 1265(C-Cstr) $\mathrm{cm}^{-1} ;{ }^{1} \boldsymbol{H}$ NMR (400 MHz, DMSO): $\delta$ ppm 2.59 (s, $\left.1 \mathrm{H},-\mathrm{CH}\right)$ 3.22-3.36 (t, 1H, -CH), 4.09-4.12 ( d, $J=9.81 \mathrm{~Hz} 1 \mathrm{H},-\mathrm{CH}), 6.29\left(\mathrm{~s}, 2 \mathrm{H}, \mathrm{NH}_{2}\right), 6.66(\mathrm{~s}, 2 \mathrm{H}$, $\left.\mathrm{NH}_{2}\right), 8.38-8.42(\mathrm{~m}, 5 \mathrm{H}, \mathrm{Ar}-\mathrm{H}), 8.64(\mathrm{~s}, 1 \mathrm{H},-\mathrm{NH}) . \mathrm{MS}: m / z=215[\mathrm{M}]^{+}$

\section{2,4-diamino-6-styryl-1,4,5,6-tetrahydropyrimidine-5-carbonitrile (PAB-102).}

IR (KBr): 3161(N-H str), 3021(CH=CH str), 3091(Ar, C-H str), 2952(C-Hstr), 2879(C-H str), 2267(C $\equiv \mathrm{N}$ str), 1609(Ar, $\mathrm{C}=\mathrm{C}$ str), 1515(Ar, $\mathrm{C}=\mathrm{C}$ str), 1508(Ar, $\mathrm{C}=\mathrm{C}$ str), 1481(C-H ben), 1425(C-H ben), 1379(C-H ben), 1252(C-C str) cm ${ }^{-1} ;{ }^{1} \boldsymbol{H}$ NMR (400 MHz, DMSO): $\delta$ ppm 2.55 $(\mathrm{s}, 1 \mathrm{H},-\mathrm{CH}) 3.29-3.37(\mathrm{t}, 1 \mathrm{H},-\mathrm{CH}), 4.05-4.18(\mathrm{dd}, J=9.79 \mathrm{~Hz} 1 \mathrm{H},-\mathrm{CH}), 5.04-5.07(\mathrm{dd}, 1 \mathrm{H}-$ $\mathrm{CH}, J=8.24), 6.32$ (s, $\left.2 \mathrm{H}, \mathrm{NH}_{2}\right), 6.6\left(\mathrm{~s}, 2 \mathrm{H}, \mathrm{NH}_{2}\right), 8.34-8.39(\mathrm{~m}, 5 \mathrm{H}, \mathrm{Ar}-\mathrm{H}), 8.74(\mathrm{~s}, 1 \mathrm{H},-\mathrm{NH})$. MS: $m / z=241[\mathrm{M}]^{+}$

\section{2,4-diamino-6-(3-chlorophenyl)-1,4,5,6-tetrahydropyrimidine-5-carbonitrile(PAB-103)}

IR (KBr): 3163(N-H str), 3097(Ar, C-H str), 2933(C-Hstr), 2877(C-H str), 2265(C $\equiv \mathrm{N}$ str), 1608(Ar, C $=\mathrm{C}$ str), 1514(Ar, C=C str), 1502(Ar, C=C str), 1489(C-H ben), 1421(C-H ben), 1371(C-H ben), 1255(C-Cstr), 744(C-Cl str) $\mathrm{cm}^{-1} ;{ }^{1} \boldsymbol{H}$ NMR (400 MHz, DMSO): $\delta$ ppm 2.61 (s, 1H, -CH) 3.30-3.34 (t, 1H, -CH), 4.07-4.09 ( d, $J=9.78 \mathrm{~Hz} 1 \mathrm{H},-\mathrm{CH}), 6.21\left(\mathrm{~s}, 2 \mathrm{H}, \mathrm{NH}_{2}\right)$, 6.68(s, 2H, NH $\left.\mathrm{N}_{2}\right), 7.82-7.87$ (t, $\left.1 \mathrm{H}, \mathrm{Ar}-\mathrm{H}\right), 8.39-8.42(\mathrm{~d}, 1 \mathrm{H}, \mathrm{Ar}-\mathrm{H}), 8.44-8.49(\mathrm{~m}, 1 \mathrm{H}, \mathrm{Ar}-\mathrm{H})$, 8.64 (s, $1 \mathrm{H},-\mathrm{NH}), 8.81(\mathrm{~s}, 1 \mathrm{H}, \mathrm{Ar}-\mathrm{H}) . \mathbf{M S}: m / z=249[\mathrm{M}]^{+}$.

2,4-diamino-6-(4-chlorophenyl)-1,4,5,6-tetrahydropyrimidine-5-carbonitrile(PAB-104). IR (KBr): 3167(N-H str), 3092(Ar, C-H str), 2942(C-Hstr), 2876(C-H str), 2260(C $\equiv \mathrm{N}$ str), 1609(Ar, C $=\mathrm{C}$ str), 1508(Ar, $\mathrm{C}=\mathrm{C}$ str), 1518(Ar, $\mathrm{C}=\mathrm{C}$ str), 1481(C-H ben), 1422(C-H ben), 1376(C-H ben), 1250(C-Cstr), 742(C-Cl str) $\mathrm{cm}^{-1} ;{ }^{1} \boldsymbol{H}$ NMR (400 MHz, DMSO): $\delta$ ppm 2.63 (s, 1H, -CH) 3.24-3.29 (t, 1H, -CH), 4.12-4.15 ( d, $J=9.78 \mathrm{~Hz} 1 \mathrm{H},-\mathrm{CH}), 6.21\left(\mathrm{~s}, 2 \mathrm{H}, \mathrm{NH}_{2}\right)$, 6.63(s, 2H, NH 2$), 7.82-7.87$ (dd, 2H, Ar-H), 8.39-8.42 (dd, 2H, Ar-H), 8.64 (s, 1H, -NH). $\boldsymbol{M S}$ : $m / z=249[\mathrm{M}]^{+}$.

\section{2,4-diamino-6-(4-fluorophenyl)-1,4,5,6-tetrahydropyrimidine-5-carbonitrile (PAB-105).}

IR (KBr): 3170(N-H str), 3094(Ar, C-H str), 2949(C-Hstr), 2875(C-H str), 2250(C $\equiv \mathrm{N}$ str), $1622(\mathrm{Ar}, \mathrm{C}=\mathrm{C}$ str), 1510(Ar, $\mathrm{C}=\mathrm{C}$ str), 1520(Ar, $\mathrm{C}=\mathrm{C}$ str), 1486(C-H ben), 1422(C-H ben), 1371(C-H ben), 1259(C-Cstr), 1065(C-F str) $\mathrm{cm}^{-1} ;{ }^{1} \boldsymbol{H}$ NMR (400 MHz, DMSO): $\delta$ ppm 2.60 (s, 1H, -CH) 3.25-3.28 (t, 1H, -CH), 4.15-4.19 ( d, $J=9.78 \mathrm{~Hz} 1 \mathrm{H},-\mathrm{CH}), 6.21\left(\mathrm{~s}, 2 \mathrm{H}, \mathrm{NH}_{2}\right)$, 6.69(s, 2H, $\left.\mathrm{NH}_{2}\right), 7.96-7.99$ (dd, 2H, Ar-H), 8.46-8.8.49 (dd, 2H, Ar-H), 8.66 (s, 1H, -NH). MS: $m / z=233[\mathrm{M}]^{+}$.

2,4-diamino-6-(4-methoxyphenyl)-1,4,5,6-tetrahydropyrimidine-5-carbonitrile(PAB-106). IR (KBr): 3170(N-H str), 3094(Ar, C-H str), 2949(C-Hstr), 2875(C-H str), 2250(C $\equiv \mathrm{N}$ str), 1619(Ar, C $=\mathrm{C}$ str), 1508(Ar, $\mathrm{C}=\mathrm{C}$ str), 1518(Ar, $\mathrm{C}=\mathrm{C}$ str), 1478(C-H ben), 1426(C-H ben), 1368 (C-H ben), 1254(C-C str), $1157\left(\mathrm{C}-\mathrm{O}-\mathrm{C}\right.$ str) $\mathrm{cm}^{-1}{ }^{1}{ }^{1} \boldsymbol{H}$ NMR (400 MHz, DMSO): $\delta$ ppm $2.67(\mathrm{~s}, 1 \mathrm{H},-\mathrm{CH}), 3.71\left(\mathrm{~s}, 3 \mathrm{H},-\mathrm{OCH}_{3}\right), 3.24-3.29(\mathrm{t}, 1 \mathrm{H},-\mathrm{CH}), 4.10-4.13(\mathrm{~d}, J=9.75 \mathrm{~Hz} 1 \mathrm{H},-$ $\mathrm{CH}), 6.23$ (s, 2H, NH $\mathrm{NH}_{2}, 6.67\left(\mathrm{~s}, 2 \mathrm{H}, \mathrm{NH}_{2}\right), 7.83-7.87$ (dd, 2H, Ar-H), 8.39-8.43 (dd, 2H, Ar-H), $8.54(\mathrm{~s}, 1 \mathrm{H},-\mathrm{NH}) . M S: m / z=245[\mathrm{M}]^{+}$. 
2,4-diamino-6-(3-nitrophenyl)- 1,4,5,6-tetrahydropyrimidine-5-carbonitrile (PAB-107). IR (KBr): 3107(N-H str), 3086 (Ar, C-H str), 3047 (C-Hstr), 1597 (Ar, C=C str), 1527 (Ar, $\mathrm{C}=\mathrm{C}$ str), 1512(Ar, $\mathrm{C}=\mathrm{C}$ str), 1479(C-H ben), 1427(C-H ben), 1315(C-H ben), 1217(C-C str) $\mathrm{cm}^{-1} ;{ }^{1} \boldsymbol{H}$ NMR (400 MHz, DMSO): $\delta$ ppm 2.56 (s, 1H, -CH) 3.20-3.24 (t, 1H, -CH), 4.06-4.08 $(\mathrm{d}, J=9.84 \mathrm{~Hz} \mathrm{1H},-\mathrm{CH}), 6.24\left(\mathrm{~s}, 2 \mathrm{H}, \mathrm{NH}_{2}\right), 6.60\left(\mathrm{~s}, 2 \mathrm{H}, \mathrm{NH}_{2}\right), 7.84-7.88$ (t, 1H, Ar-H), 8.35$8.37(\mathrm{~d}, 1 \mathrm{H}, \mathrm{Ar}-\mathrm{H}), 8.44-8.47(\mathrm{~m}, 1 \mathrm{H}, \mathrm{Ar}-\mathrm{H}), 8.63(\mathrm{~s}, 1 \mathrm{H},-\mathrm{NH}), 8.83(\mathrm{~s}, 1 \mathrm{H}, \mathrm{Ar}-\mathrm{H}) . \boldsymbol{M S}: m / z=$ $260[\mathrm{M}]^{+}$.

\section{2,4-diamino-6-(4-hydroxy-3-methoxyphenyl)-1,4,5,6-tetrahydropyrimidine-5carbonitrile (PAB-108).}

IR (KBr): 3480(OH str), 3164(N-H str), 3023 (Ar, C-H str), 2947(C-H str), 2822(C-H str), 2241(C $\equiv \mathrm{N}$ str), 1606(Ar, $\mathrm{C}=\mathrm{C}$ str), 1523(Ar, $\mathrm{C}=\mathrm{C}$ str), 1538(Ar, $\mathrm{C}=\mathrm{C}$ str), 1482(C-H ben), 1357(C-H ben), 1330(C-H ben), 1230(C-C str), 1078(C-O-C str), $\mathrm{cm}^{-1} ;{ }^{1} \boldsymbol{H}$ NMR $(400 \mathrm{MHz}$, DMSO): $\delta$ ppm $2.50(\mathrm{~s}, 1 \mathrm{H},-\mathrm{CH}) 3.29-3.33(\mathrm{t}, 1 \mathrm{H},-\mathrm{CH}), 3.74\left(\mathrm{~s}, 3 \mathrm{H}, \mathrm{OCH}_{3}\right), 4.07-4.09(\mathrm{~d}, J=$ $9.86 \mathrm{~Hz} 1 \mathrm{H},-\mathrm{CH}), 4.89(\mathrm{~s}, 1 \mathrm{H},-\mathrm{OH}), 6.26\left(\mathrm{~s}, 2 \mathrm{H}, \mathrm{NH}_{2}\right), 6.65\left(\mathrm{~s}, 2 \mathrm{H}, \mathrm{NH}_{2}\right), 7.84-7.88(\mathrm{t}, 1 \mathrm{H}, \mathrm{Ar}-$ H), 8.35-8.37 (d, 1H, Ar-H), 8.45-8.49 (m, 1H, Ar-H), 8.69 (s, 1H, -NH), MS: $m / z=261[\mathrm{M}]^{+}$.

\section{2,4-diamino-6-(furan -2-yl)- 1,4,5,6-tetrahydropyrimidine-5-carbonitrile (PAB-109).}

IR (KBr): 3124(N-H str), 3043(Ar, C-H str), 2922(C-H str), 2847(C-H str), 2245(C $\equiv N$ str), 1606(Ar, C $=\mathrm{C}$ str), 1552(Ar, $\mathrm{C}=\mathrm{C}$ str), 1529(Ar, $\mathrm{C}=\mathrm{C}$ str), 1456(C-H ben), 1394(C-H ben), 1330(C-H ben), 1230(C-C str), 1070(C-O-C str), 1022(C-O-C str) $\mathrm{cm}^{-1} ;{ }^{1} \boldsymbol{H}$ NMR $(400 \mathrm{MHz}$, DMSO): $\delta$ ppm $2.57(\mathrm{~s}, 1 \mathrm{H},-\mathrm{CH}) 3.32-3.35(\mathrm{t}, 1 \mathrm{H},-\mathrm{CH}), 4.07-4.09(\mathrm{~d}, J=9.80 \mathrm{~Hz} 1 \mathrm{H},-\mathrm{CH})$, $6.18\left(\mathrm{~s}, 2 \mathrm{H}, \mathrm{NH}_{2}\right), 6.64\left(\mathrm{~s}, 2 \mathrm{H}, \mathrm{NH}_{2}\right), 7.84-7.88(\mathrm{~s}, 1 \mathrm{H}, \mathrm{Ar}-\mathrm{H}), 8.35-8.37$ (s, 1H, Ar-H), 8.45$8.49(\mathrm{~s}, 1 \mathrm{H}, \mathrm{Ar}-\mathrm{H}), 8.71(\mathrm{~s}, 1 \mathrm{H},-\mathrm{NH})$,

MS: $m / z=205[\mathrm{M}]^{+}$.

2,4-diamino-6-(4-hydroxyphenyl)-1,4,5,6-tetrahydropyrimidine-5-carbonitrile (PAB-110). IR (KBr): 3635(OH str), 3165(N-H str), 3088(Ar, C-H str), 2942(C-H str), 2865(C-H str), 2250( $\mathrm{C} \equiv \mathrm{N}$ str), $1619(\mathrm{Ar}, \mathrm{C}=\mathrm{C}$ str), $1510(\mathrm{Ar}, \mathrm{C}=\mathrm{C}$ str), 1528(Ar, $\mathrm{C}=\mathrm{C}$ str), 1482(C-H ben), 1426(C-H ben), 1371(C-H ben), 1259(C-C str), 1210 (C-O str) cm ${ }^{-1} ;{ }^{1} \boldsymbol{H}$ NMR (400 MHz, DMSO): $\delta$ ppm 2.69 (s, $1 \mathrm{H},-\mathrm{CH}), 3.29-3.32(\mathrm{t}, 1 \mathrm{H},-\mathrm{CH}), 4.15-4.18(\mathrm{~d}, J=9.78 \mathrm{~Hz} 1 \mathrm{H},-\mathrm{CH})$, 4.64(s, $1 \mathrm{H}, \mathrm{OH}), 6.28\left(\mathrm{~s}, 2 \mathrm{H}, \mathrm{NH}_{2}\right), 6.69\left(\mathrm{~s}, 2 \mathrm{H}, \mathrm{NH}_{2}\right), 7.85-7.89$ (dd, $\left.2 \mathrm{H}, \mathrm{Ar}-\mathrm{H}\right), 8.46-8.50$ (dd, $2 \mathrm{H}, \mathrm{Ar}-\mathrm{H}), 8.59$ (s, $1 \mathrm{H},-\mathrm{NH}) . \mathbf{M S}: m / z=231[\mathrm{M}]^{+}$.

\section{1. Instrumentation}

Thermo gravimetric analysis (TGA) and Differential Scanning Calorimetry (DSC) measurements were made on the instrument "Pyris-1, Perkin Elmer Thermal Analysis" at the heating rate of $10{ }^{\circ} \mathrm{C} / \mathrm{min}$ in nitrogen atmosphere for all the tetrahydropyrimidine derivatives.

\section{RESULTS AND DISCUSSION}

Figure 1 shows the TGA thermo grams of PAB-101. Various thermal properties such as initial decomposition temperature (IDT), the decomposition temperature range and the maximum degradation along with the percentage weight loss are reported in Table 2. 
Table 2. TGA data for synthesized tetrahydropyrimidine derivatives.

\begin{tabular}{|c|c|c|c|c|c|c|c|}
\hline $\begin{array}{c}\text { Comp. } \\
\text { Code }\end{array}$ & $\begin{array}{c}\text { Amount } \\
\mathbf{( m g . )}\end{array}$ & $\begin{array}{c}\text { Initial } \\
\text { Decomp. } \\
\mathbf{T e m p} \\
\left({ }^{\circ} \mathbf{C}\right)\end{array}$ & $\begin{array}{c}\text { Decomp. } \\
\text { Range }\left({ }^{\circ} \mathbf{C}\right)\end{array}$ & $\begin{array}{c}\text { \% } \\
\mathbf{W t} \\
\text { loss }\end{array}$ & $\begin{array}{c}\text { Residual } \\
\mathbf{w t .} \\
\text { loss }(\mathbf{m g .})\end{array}$ & $\begin{array}{c}\text { Max. } \\
\text { Degrad. } \\
\text { Temp. } \\
\left({ }^{\circ} \mathbf{C}\right)\end{array}$ & Transition \\
\hline PAB-101 & 2.297 & 110.00 & $110-250$ & 99.02 & 2.275 & 168.08 & Endo \\
\hline PAB-102 & 5.104 & 70.00 & $70-296.50$ & 49.72 & 2.538 & 191.49 & Endo \\
\hline PAB-103 & 2.497 & 80.00 & $80-190$ & 88.76 & 2.217 & 164.67 & Endo \\
\hline PAB-104 & 1.741 & 140.00 & $140-230$ & 93.72 & 1.632 & 181.74 & Endo \\
\hline PAB-105 & 2.160 & 110.00 & $110-230$ & 99.85 & 2.157 & 172.27 & Endo \\
\hline PAB-106 & 2.938 & 150.00 & $150-246.20$ & 71.26 & 2.094 & 209.00 & Endo \\
\hline PAB-107 & 3.127 & 153.51 & $153-296.30$ & 88.43 & 2.766 & 247.22 & Endo \\
\hline PAB-108 & 1.071 & 90.00 & $90-246.80$ & 81.37 & 0.872 & 210.00 & Endo \\
\hline PAB-109 & 2.969 & 100.00 & $100-240$ & 92.72 & 2.753 & 166.72 & Endo \\
\hline PAB-110 & 1.903 & 145.45 & $145.45-$ & 87.17 & 1.659 & 213.18 & Endo \\
\hline
\end{tabular}

For all the compounds, degradation is single step process. Out of ten compounds, PAB102 is most unstable which is followed by PAB-103. PAB-107 is the most stable compound which is followed by PAB-106. All the studied compounds have the same central moiety but substitution groups are different.

Thus, substitution affects the thermal stability of a compound. PAB-102 contains cinnamaldehyde as substitution to aromatic ring. Thus, cinnamaldehyde makes the compound unstable whereas chloro group at meta position (as in PAB-103) makes it a little bit less stable. However, the presence of nitro group at meta position increases the stability as is the case for PAB-107.

Whereas presence of methoxy group at para position decreases the stability slightly. Further, it is observed that the position of group affect the stability as observed in case of PAB-103 and PAB-104. Both these compounds contain chloro group. In PAB-103, it is at meta position whereas in PAB-104, it is at para position.

It is observed that when chloro group is at meta position, it decreases the stability in comparison to that at para position. Both methoxy and hydroxyl groups when present alone at para position increases the stability as observed in PAB-106 and PAB-110. However, when both groups are present together as in PAB-108, stability is reduced.

Further, it is observed that although PAB-102 is most unstable, weight loss is minimum for this compound whereas for PAB-101, it is maximum. The variation in thermal decomposition may also be due to some intermolecular interactions (structural as well as electronic). 


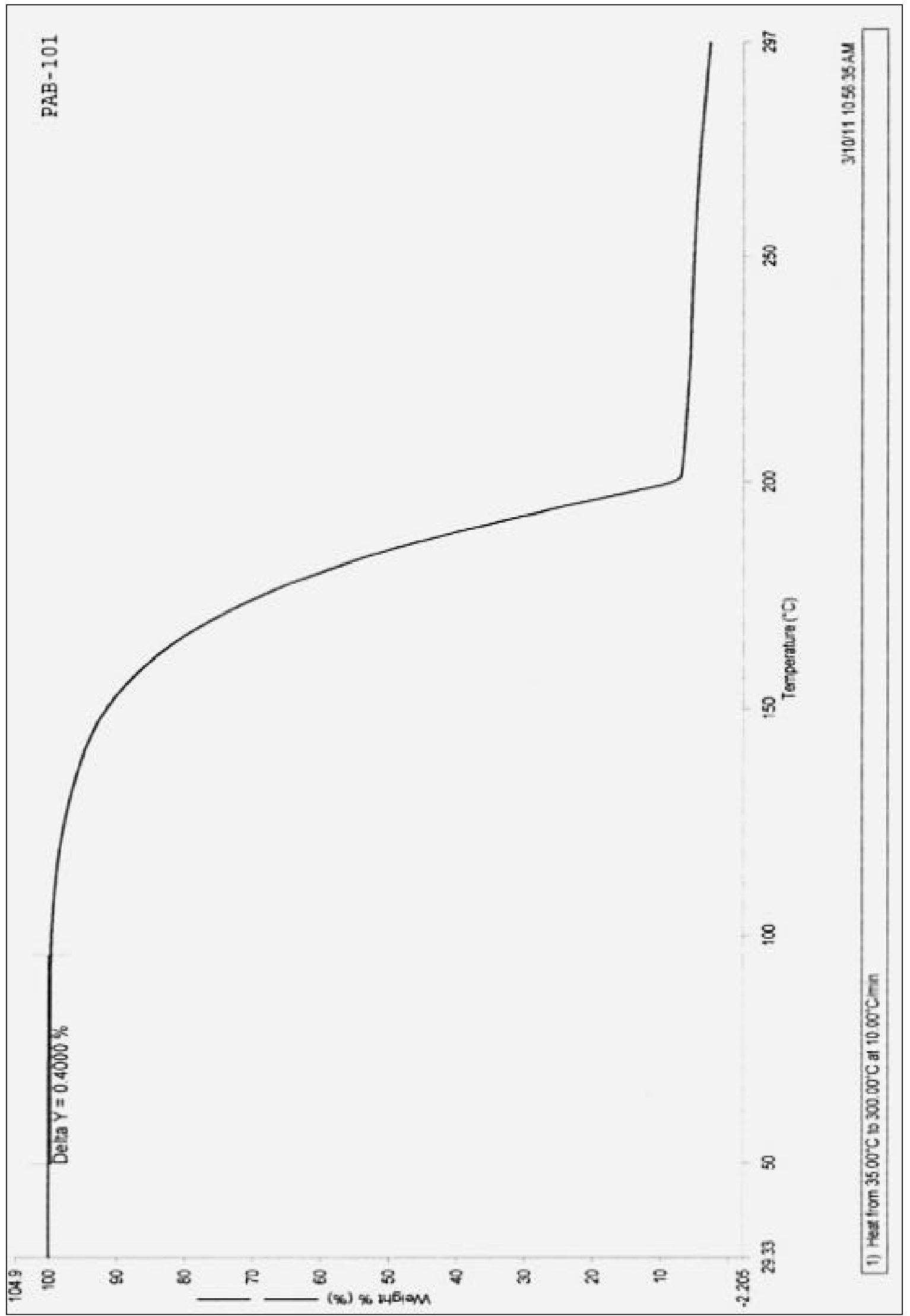

Figure 1. Themogram of PAB-101. 
Further, various kinetic parameters, such as order of the degradation $(n)$, energy of activation $(E)$, frequency factor $(A)$ and entropy change $(\Delta S)$ have also been calculated from the thermograms.

The order of the degradation $(n)$ and energy of activation $(E)$ are calculated using Freeman and Carroll relation ${ }^{19}$

$$
\frac{\Delta \log d w / d t}{\Delta \log W_{r}}=n-\frac{E}{2.303 R} \cdot \frac{\Delta\left(\frac{1}{T}\right)}{\Delta \log W_{r}}
$$

where $W_{r}=W_{C}-w . W_{C}$ is weight loss at the completion of reaction and $w$ is the total weight loss up to time $t$.

A plot of left hand side against $\Delta(1 / T) /\left(\Delta \log W_{r}\right)$ gives a straight line with a slope equal to $-E / 2.303 R$ and the intercept is equal to $n$.

The frequency factor $A$ and entropy change $\Delta S$ can be determined by the following equations:

$$
\begin{gathered}
\ln E-\ln \left(R T_{S}{ }^{2}\right)=\ln A-\ln \beta-E /\left(R T_{S}\right) \\
A=\left(k_{b} T / h\right) e^{\Delta S / R}
\end{gathered}
$$

where $k_{b}$ is Boltzmann constant and $h$ is Planck's constant.

The evaluated kinetic parameters are reported in Table 3 . The order of reaction $(n)$ varies from 0.64 to 8.09 . The value of $n$ is minimum for PAB-104 and maximum for PAB103.

Table 3. The kinetic parameters of tetrahydropyrimidine derivatives.

\begin{tabular}{|c|c|c|c|c|}
\hline $\begin{array}{c}\text { Comp. } \\
\text { code }\end{array}$ & $\mathbf{n}$ & $\begin{array}{c}\mathbf{E} \\
(\mathbf{k J})\end{array}$ & $\begin{array}{c}\mathbf{A} \\
\left.\mathbf{( S e c}^{-1}\right)\end{array}$ & $\begin{array}{c}\Delta \mathbf{S}^{\mathbf{0}} \\
\left.\mathbf{( J}^{-1}\right)\end{array}$ \\
\hline PAB-101 & 2.89 & 418.46 & $4.47 \times 10^{49}$ & 855.48 \\
\hline PAB-102 & 2.79 & 434.24 & $7.52 \times 10^{48}$ & 840.22 \\
\hline PAB-103 & 8.09 & 251.64 & $7.88 \times 10^{29}$ & 477.38 \\
\hline PAB-104 & 0.64 & 529.69 & $9.75 \times 10^{60}$ & 1072.28 \\
\hline PAB-105 & 1.78 & 535.92 & $1.09 \times 10^{63}$ & 1111.64 \\
\hline PAB-106 & 5.14 & 460.97 & $9.85 \times 10^{49}$ & 861.30 \\
\hline PAB-107 & 3.29 & 253.15 & $1.34 \times 10^{25}$ & 384.64 \\
\hline PAB-108 & 1.32 & 480.17 & $9.59 \times 10^{51}$ & 899.36 \\
\hline PAB-109 & 4.44 & 401.11 & $5.06 \times 10^{47}$ & 818.24 \\
\hline PAB-110 & 2.44 & 490.65 & $5.91 \times 10^{52}$ & 914.42 \\
\hline
\end{tabular}


The energy of activation $(E)$ is highest for PAB-105 containing fluoro group at para position and minimum for $\mathrm{PAB}-103$ which is containing chloro group at meta position. The frequency factor $(A)$ is also highest for PAB-105 but minimum for PAB-107.

Further, change in entropy $\left(\triangle S^{0}\right)$ for all these reactions were also calculated by equation and are reported in Table 3. The entropy change $\left(\Delta S^{0}\right)$ is found to be positive for all ten compounds which indicate that the transition state is less ordered than the original compound ${ }^{20}$.

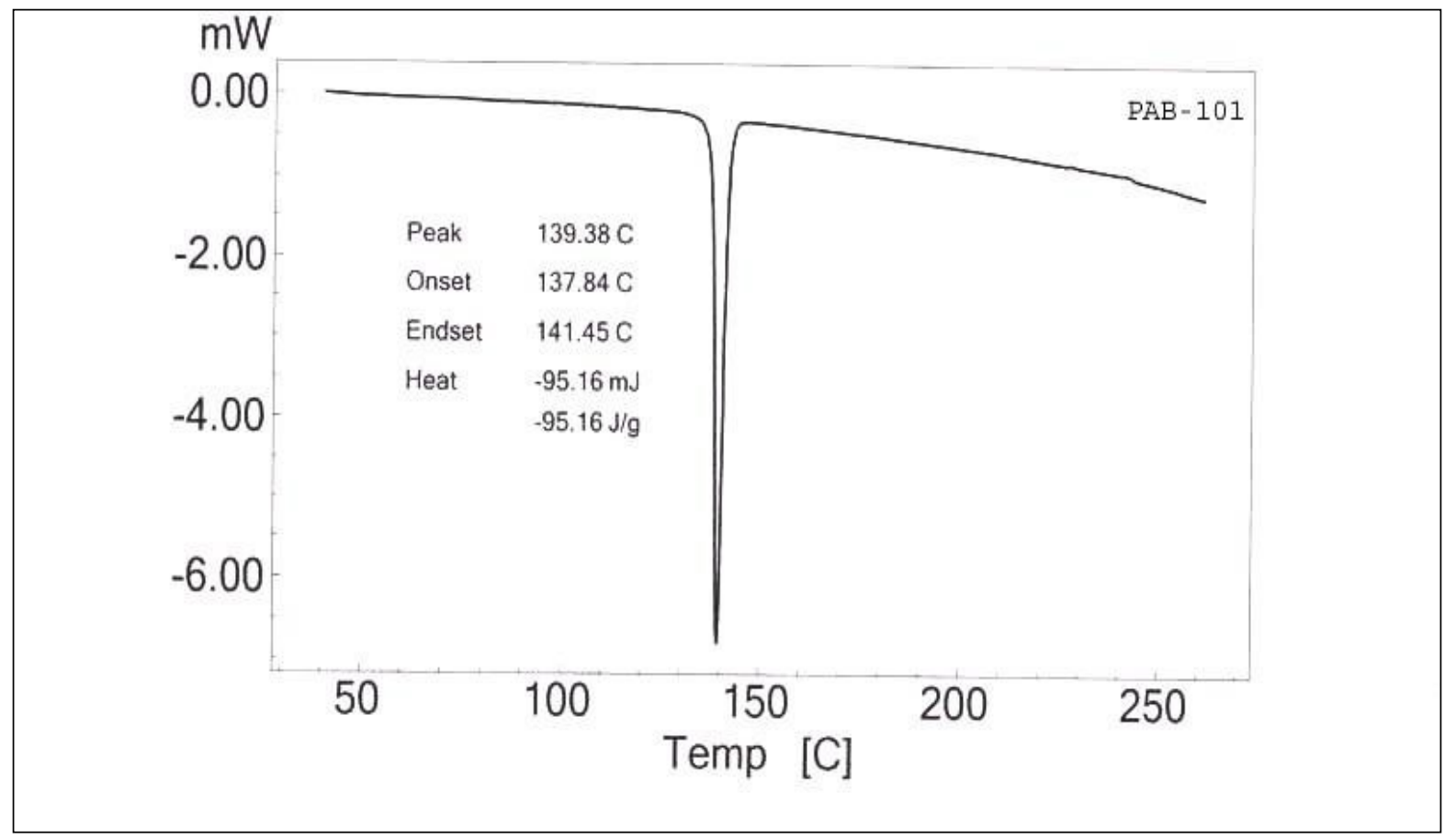

Figure 2. The DSC graph of PAB-101.

Figure 2 shows DSC of PAB-101. From DSC, melting points of all the compounds are determined and are given in Table 4 along with melting points determined by open capillary method.

There is good agreement between the values evaluated from DSC and those determined by open capillary method.

Table 4. The melting temperature $\left({ }^{\circ} \mathrm{C}\right)$ of tetrahydropyrimidine derivatives by DSC and open capillary method.

\begin{tabular}{|c|c|c|c|c|c|c|c|c|c|c|}
\hline $\begin{array}{c}\text { Comp. } \\
\text { Code }\end{array}$ & $\begin{array}{c}\text { PAB- } \\
\mathbf{1 0 1}\end{array}$ & $\begin{array}{c}\text { PAB- } \\
\mathbf{1 0 2}\end{array}$ & $\begin{array}{c}\text { PAB- } \\
\mathbf{1 0 3}\end{array}$ & $\begin{array}{c}\text { PAB- } \\
\mathbf{1 0 4}\end{array}$ & $\begin{array}{c}\text { PAB- } \\
\mathbf{1 0 5}\end{array}$ & $\begin{array}{c}\text { PAB- } \\
\mathbf{1 0 6}\end{array}$ & $\begin{array}{c}\text { PAB- } \\
\mathbf{1 0 7}\end{array}$ & $\begin{array}{c}\text { PAB- } \\
\mathbf{1 0 8}\end{array}$ & $\begin{array}{c}\text { PAB- } \\
\mathbf{1 0 9}\end{array}$ & $\begin{array}{c}\text { PAB- } \\
\mathbf{1 1 0}\end{array}$ \\
\hline DSC & 140.00 & 113.29 & 153.75 & 163.51 & 129.04 & 116.48 & 105.77 & 132.94 & 71.60 & 177.94 \\
\hline $\begin{array}{c}\text { Open } \\
\text { capillary }\end{array}$ & 142 & 114 & 154 & 164 & 129 & 116 & 106 & 132 & 72 & 178 \\
\hline
\end{tabular}




\section{CONCLUSION}

For the studied compounds, degradation is single step process with different order of reaction. The thermal stability depends upon the type of substituent present.

\section{References}

1. Mashkovskii, D.; Medicinals [in Russian], 12th ed., Meditsina, Moscow 1993, Part 1, p. 736; 1993, Part 2, p. 688.

2. Nikolaeva, B.; (ed.), Medicinal Products from Foreign Firms in Russia [in Russian], Astrafarmservis, Moscow, (1993), p. 720.

3. Bahekar, S.; Shinde, D.; Bioorganic and Medicinal Chemistry Letters 14(7) (2004) 17331736.

4. Mokale, S.; Shinde, S.; Elgire, R.; Sangshetti, J. and Shinde, D.; Bioorganic and Medicinal Chemistry Letters 20(15) (2010) 4424-4426.

5. Tozkoparan, B.; Ertan, M.; Kelicen, P., Demirdamar, R.; Farmaco. 54(9) (1999) 588-593.

6. Arora, N.; Pandeya, S.; International Journal of Pharmaceutical Sciences and Research 11(1) (2011) 48-52.

7. Linsheng, L.; Guibao, Z.; Chuanlin, Y.; Qun, Z. and Nana, C.; Faming Zhuanli Shenqing (2011), CN 102228461 A 20111102

8. Li, Baoqiu; Li, Lingzi; Ji, Gao.; Faming Zhuanli Shenqing (2011), CN 102274238 A 20111214.

9. Ji-Xin, Y.; Xiao-Qing, C.; Di-Mei, C., and Mao-Lin, H.; Chinese Journal of Chemistry 25(3) (2007) 417-421.

10. Weinhardt, K.; Wallach, M.B.; and Marx, M.; Journal of Medicinal Chemistry 28(6) (1985) 694-698.

11. Champaneri, H.R.; Modi, S.R.; and Naik, H.B.; Asian Journal of Chemistry 6(3) (1994) 737-738.

12. Satyavathi, K.; Naga Ravi, K.T.; Bhoja, R.P. and Sharmila, M.; Asian Journal of Chemistry 22(7) (2010) 5182-5186.

13. Basavaraja, H. S.; Basavaraj, P.; Vijaykumar, M.; Hussain, M. M. and Chidananda, B. N.; Indian journal of heterocylic 20(3) (2011) 237-240.

14. D. Giron, Journal of pharmaceutical and biomedical analysis 4(6) (1986) 755-770.

15. D. Giron, "Thermal Analysis of Drugs and Drug Products" Encyclopedia of Pharmaceutical Technology, Third Edition, (2006).

16. Hardy, M. J.; Charsley E. L. and Warrington, S. B.; Thermal analysis-techniques and applications, The Royal Society of Chemistry, Cambridge (1992), 180-197.

17. Rubinstein M. H.; "Pharmaceutical technology: drug stability." Chichester: Ellis Horwood; (1989). 
18. Brittain H G.; "Physical characterization of pharmaceutical solids." New York: Marcel Dekker, Inc.; (1995).

19. Freeman, E.S. and Carroll, B.; Journal of physical chemistry 62 (1958) 394-397.

20. Mishra, A.P.; Tiwari, V.; Singhal, R. and Gautam, S.K.; Indian Journal of Chemistry. 41A(10) (2002) 2092-2095. 